\title{
The Multisection Of Any Arbitrary Angle With Straightedge And Compass Only
}

\author{
Hemendra Kumar Srivastava \\ M.Sc. Maths (in 1973), H. No. N 8/256-D 3 K, Jeewan Nagar Colony, Newada, Sunderpur, Varanasi-221005, \\ U.P. (India).
}

\section{Preface}

At school level students learn about bisection of any arbitrary angle or repeated bisections if required with the help of straightedge and compass only. They do not come across about angle trisection or other higher multisections with the help of only these tools.In 1837 P. Wantzel a French mathematician proved the impossibility of trisection of any arbitrary angle with only stranghtedge and compass except some specified angles.In my present work I have endeavoured to demostrate methods for drawing very approximate multisections of any arbitrary angles lying between 0 to $90^{\circ}$ with the help of only stranghtedge and compass. I came to know about impossibility of angle trisection at my B.Sc. standard in 1970 through a mathematical journal and since then I was working on it. Thus my present work is the result of many years of dedicated efforts.

I. Multisection of an arbitrary angle with the help of straightedge and compass only

1. Before proceeding further we shall first derive the formula or results (4) \& (5) worked out in the following pages.

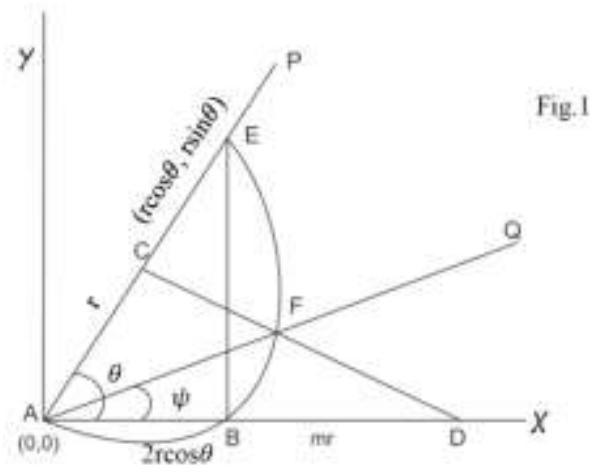

Given $\mathrm{AX} \& \mathrm{AY}$ are $\mathrm{X} \& \mathrm{Y}$ axis.

$\mathrm{AP}$ a line making an angle $\theta$ with $\mathrm{X}$ axis such that $\left(0^{0}<\theta \leq 90^{\circ}\right)$

$\mathrm{C}$ any point on the line $\mathrm{AP}, \mathrm{a}$ semi-circle $\mathrm{ABFE}$ is drawn taking $\mathrm{C}$ as centre and $\mathrm{AC}$ as radius $r$.

The semi circle cuts $\mathrm{X}$ axis on pt. $\mathrm{B}$. AE is diameter.

$\mathrm{BD}$ a length is cut equal to $\mathrm{mr}$ ( $\mathrm{m}$ times of radius $\mathrm{r}$ ). points $\mathrm{C} \& \mathrm{D}$ are joined.

To find the locus of point $F$ where the line $C D$ cuts the semicircle at $F$.

Point $C$ is $(r \cos \theta, r \sin \theta)$

Point $\mathrm{E}$ is $(2 \mathrm{r} \cos \theta, 2 \mathrm{r} \sin \theta)$

So length $\mathrm{A} B$ is $=2 \mathrm{r} \cos \theta \& \mathrm{BD}=\mathrm{mr}$

Hence coordinates of pt. D is $(2 r \cos \theta+m r, o)$

So equation of line $\mathrm{CD}$ is

$\mathrm{y}-\mathrm{y}_{1}=\frac{y_{2}-y_{1}}{x_{2}-x_{1}}\left(\mathrm{x}-\mathrm{x}_{1}\right)$

Or $\mathrm{y}-\mathrm{r} \sin \theta=\frac{0-r \sin \theta}{2 r \cos \theta+m r-r \cos \theta}(\mathrm{x}-\mathrm{r} \cos \theta)$

Or $y-r \sin \theta=\frac{-r \sin \theta}{r \cos \theta+m r}(x-r \cos \theta)$

Or $(\mathrm{y}-\mathrm{r} \sin \theta)(\mathrm{r} \cos \theta+m r)=\mathrm{r}^{2} \sin \cos \theta-x r \operatorname{Sin} \theta$

Or $(\mathrm{y}-\mathrm{r} \sin \theta) \times r(\cos \theta+m)=r(r \sin \theta \cos \theta-x \sin \theta)$

Or $(y-r \sin \theta)(\cos \theta+m)=r \sin \theta \cos \theta-x \sin \theta$

Or $y \cos \theta+m y-r \sin \theta \cos \theta=r \sin \theta \cos \theta-x \sin \theta+m r \sin \theta$ 
Or $y \cos \theta+m y+x \sin \theta=2 r \sin \theta \cos \theta+m r \sin \theta$

Now equation of the circle is

$\left(\mathrm{x}-\mathrm{x}_{1}\right)^{2}+\left(\mathrm{y}-\mathrm{y}_{1}\right)^{2}=\mathrm{r}^{2}$

$=(x-r \cos \theta)^{2}+(y-r \sin \theta)^{2}=r^{2}$

$=\mathrm{x}^{2}+\mathrm{r}^{2} \cos ^{2} \theta-2 \mathrm{xrcos} \theta+\mathrm{y}^{2}+\mathrm{r}^{2} \sin ^{2} \theta-2 \mathrm{yr} \sin \theta=\mathrm{r}^{2}$

Or $\mathrm{x}^{2}+\mathrm{y}^{2}+\mathrm{r}^{2}\left(\cos ^{2} \theta+\sin ^{2} \theta\right)-2 \mathrm{r}(\mathrm{x} \cos \theta+\mathrm{y} \sin \theta)=\mathrm{r}^{2}$

Or $x^{2}+y^{2}+r^{2}-2 r(x \cos \theta+y \sin \theta)=r^{2}$

Or $\mathrm{x}^{2}+\mathrm{y}^{2}=2 \mathrm{r}(\mathrm{x} \cos \theta+\mathrm{y} \sin \theta)$

Now from (1) $r=\frac{y \cos \theta+x \sin \theta+m y}{2 \sin \theta \cos \theta+m \sin \theta}$

Putting this value of $r$ in eq. (3) we have

$\mathrm{x}^{2}+\mathrm{y}^{2}=\frac{2(y \cos \theta+x \sin \theta+m y)(x \cos \theta+y \sin \theta)}{2 \sin \theta \cos \theta+m \sin \theta}$

or $\left(\mathrm{x}^{2}+\mathrm{y}^{2}\right)(2 \sin \theta \cos \theta+m \sin \theta)$

$=2(y \cos \theta+x \sin \theta+m y)(x \cos \theta+y \sin \theta)$

L.H.S. $=\quad 2 x^{2} \sin \theta \cos \theta+x^{2} m \sin \theta+2 y^{2} \sin \theta \cos \theta+m y^{2} \sin \theta$

R.H.S. $=2 y x \cos ^{2} \theta+2 y^{2} \sin \theta \cos \theta+2 x^{2} \sin \theta \cos \theta+2 x y \sin ^{2} \theta+$

$2 x y m \cos \theta+2 m y^{2} \sin \theta$

$2 x y m \cos \theta+2 m y^{2} \sin \theta$

$$
=\quad 2 x y\left(\cos ^{2} \theta+\sin ^{2} \theta\right)+2 y^{2} \sin \theta \cos \theta+2 x^{2} \sin \theta \cos \theta+
$$

Simplifying LHS \& RHS we get:-

$m x^{2} \sin \theta+m y^{2} \sin \theta=2 x y+2 x y m \cos \theta+2 m y^{2} \sin \theta$

OR $m x^{2} \sin \theta=2 m y^{2} \sin \theta-m y^{2} \sin \theta+2 x y m \cos \theta+2 x y$

OR $m y^{2} \sin \theta+2 x y(1+m \cos \theta)-m x^{2} \sin \theta=0$

OR $y=\frac{-2 x(1+m \cos \theta) \pm \sqrt{4 x^{2}(1+m \cos )^{2}+4 m^{2} x^{2} \sin ^{2} \theta}}{2 m \sin \theta}$

By solving for $y$ -

OR $y=\frac{-2 x(1+m \cos \theta) \pm \sqrt{4 x^{2}\left(1+m^{2} \cos ^{2} \theta+2 m \cos \theta+4 m^{2} x^{2} \sin ^{2} \theta\right.}}{2 m \sin \theta}$

$$
=\frac{-2 x(1+m \cos \theta) \pm \sqrt{4 x^{2}\left\{1+\left(m^{2} \cos ^{2} \theta+m^{2} \sin ^{2} \theta\right)+2 m \cos \theta\right\}}}{2 m \sin \theta}
$$

OR $y=\frac{-2 x(1+m \cos \theta) \pm \sqrt{4 x^{2}\left(1+m^{2}+2 m \cos \theta\right)}}{2 m \sin \theta}$

$$
=\frac{-2 x(1+m \cos \theta) \pm 2 x \sqrt{1+m^{2}+2 m \cos \theta}}{2 m \sin \theta}
$$

OR $y=\frac{x \sqrt{1+m^{2}+2 m \cos \theta}-x(1+m \cos \theta)}{m \sin \theta}$

Only taking $(+)$ sign. $\left(\right.$ since $\left.0^{0}<\theta \leq 90^{\circ}\right)$

$$
=x\left\{\frac{\sqrt{1+m^{2}+2 m \cos \theta}-(1+m \cos \theta)}{m \sin \theta}\right\}
$$

Hence Locus of the point $F$, the point of intersection of line CD \& circle ABFE is a straight line passing through origin $\mathrm{A}(0,0)$ and making an angle $\psi$ with $\mathrm{X}$ axis.

such that,

$\tan \psi=\frac{\sqrt{1+m^{2}+2 m \cos \theta}-(1+m \cos \theta)}{m \sin \theta}$

which is independent of $r$ and a function of $\theta \& m$ only.

This is an important result and the basis of angle multisections.

Note that the length BD is $=\mathrm{m} \times \mathrm{r}$

that is $m$ times or radius $r$

if BD is $\frac{m}{n}$ times of $r$ then (4) becomes

$\tan \psi=\frac{\sqrt{m^{2}+n^{2}+2 m n \cos \theta}-(n+m \cos \theta)}{m \sin \theta}$.

1.1 Corollary-1

if $\mathrm{BD}=\mathrm{mr}$ and $\mathrm{m}=1$

then $\mathrm{BD}=\mathrm{r}$ so from $(4)$

$\tan \psi=\frac{\sqrt{1^{2}+1^{2}+2 \cdot 1 \cos \theta}-(1+\cos \theta)}{\sin \theta}$

or $=\frac{\sqrt{2+2 \cos \theta}-(1+\cos \theta)}{\sin \theta}$ 
$=\frac{\sqrt{2(1+\cos \theta)}-(1+\cos \theta)}{\sin \theta}$

$=\frac{\sqrt{2.2 \cos ^{2} \frac{\theta}{2}}-2 \cos ^{2} \frac{\theta}{2}}{2 \sin \frac{\theta}{2} \cos \frac{\theta}{2}}$

$=\frac{2 \cos \frac{\theta}{2}-2 \cos \frac{2}{2} \frac{\theta}{2}}{2 \sin \frac{\theta}{2} \cos \frac{\theta}{2}}$

$=\frac{2 \cos \frac{\theta}{2}\left(1-\cos \frac{\theta}{2}\right)}{2 \sin \frac{\theta}{2} \cos \frac{\theta}{2}}$

$=\frac{1-\cos \frac{\theta}{2}}{\sin \frac{\theta}{2}}=\frac{2 \sin ^{2} \frac{\theta}{4}}{2 \sin \frac{\theta}{4} \cos \frac{\theta}{4}}$

$=\frac{\sin \frac{\theta}{4}}{\cos \frac{\theta}{4}}=\tan \frac{\theta}{4}$

So $\tan \psi=\tan \frac{\theta}{4}$

Or $\psi=\frac{\theta}{4}$

Hence if length $\mathrm{BD}=\mathrm{r}$ (radius) then

$\angle Q A D=\frac{1}{4} \angle P A D=\frac{\theta}{4}$

This is another important result as it gives us a method for constructing $\frac{1}{4}^{\text {th }}$ of any original angle without bisecting it with the help of straightedge and compass only.

In Fig. 2

$\angle E A D=\theta, m=1$

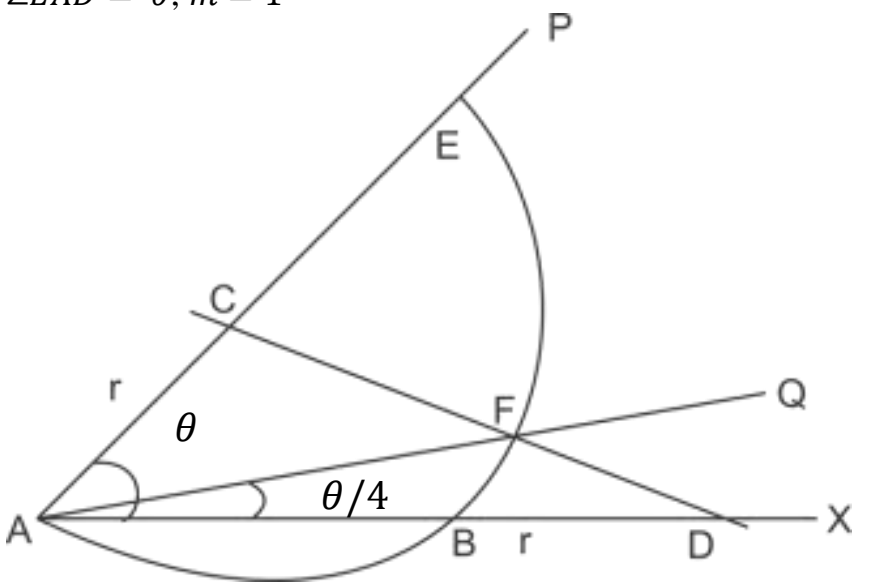

Fig. 2

$m=1$

$B D=A C=r$

By corollary $1 \angle F A D=\frac{1}{4} \theta$

\subsection{Corollary-2}

If $\mathrm{D}$ proceeds further \& $\mathrm{BD}$ becomes larger $\angle F A D$ increases gradually and finally when $\mathrm{BD}$ is infinite line $\mathrm{CD}$ becomes parallel to $\mathrm{AD}$ or $\mathrm{X}$ axis then equation of line $\mathrm{CD}$ becomes.

$y=r \sin \theta$

and eq. of circle is $(x-r \cos \theta)^{2}+(y-r \sin \theta)^{2}=r^{2}$

or $(x-r \cos \theta)^{2}-0^{2}=r^{2}$

or $\quad\left(x-\frac{y \cos \theta}{\sin \theta}\right)^{2}=\left(\frac{y}{\sin \theta}\right)^{2}$

or $\quad x-\frac{y \cos \theta}{\sin \theta}=\frac{y}{\sin \theta}$

or $\quad x \sin \theta-y \cos \theta=y$

or $\quad x \sin \theta=y(1+\cos \theta)$

or $\quad \mathrm{y} \times 2 \cos ^{2} \frac{\theta}{2}=x \times 2 \sin \frac{\theta}{2} \cos \frac{\theta}{2}$

or $\quad \mathrm{y} \times \cos \frac{\theta}{2}=x \times \sin \frac{\theta}{2}$

or $\quad y=x \cdot \tan \frac{\theta}{2}$

or $\tan \psi=\tan \frac{\theta}{2}$ 
or $\quad \psi=\frac{\theta}{2}$

So in this case the line AF bisects the angle $\mathrm{E}$ A B or $\theta$.

So this corollary gives us another method of bisecting any given angle.

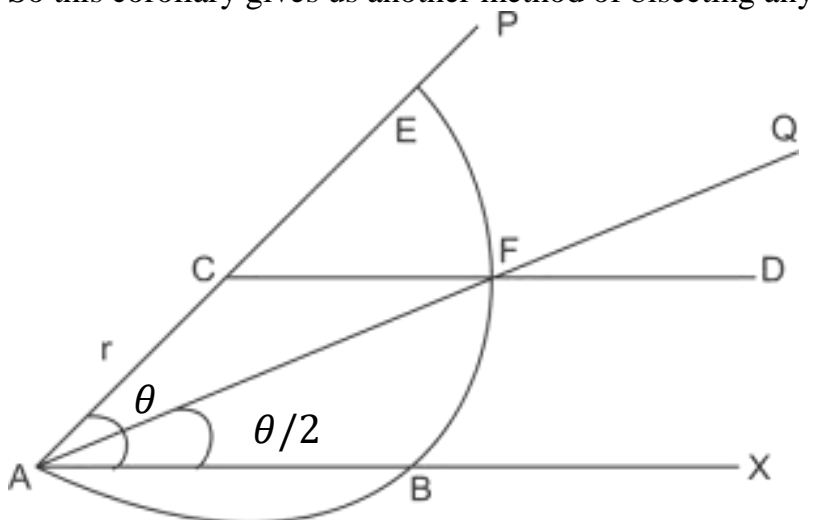

Fig. 3

$m=\infty$

Line $\mathrm{CD}$ is parallel to $\mathrm{AX}$

$\angle F A B=\frac{1}{2} \angle E A X=\frac{\theta}{2}$

When the length $\mathrm{BD}=0$, pt. F concides with pt. $\mathrm{B}$ and $\angle F A B=0$ and as per corollary 2 when BD is infinite $\angle F A B=\frac{1}{2} \angle E A B$

So by taking different values of $m$ we can multisect the given angle $\theta$ with the help of straight edge and compass only.

\section{Some results of taking different values of $m$ and $\theta$ and $\psi$}

A for trisection of an angle

\begin{tabular}{|l|l|l|}
\hline \multicolumn{1}{|c|}{$\theta$} & $m$ or $\frac{m}{n}$ & \\
\hline $90^{0}$ & \multicolumn{1}{|c|}{$\sqrt{3}$} & $30^{0}$ exactly \\
\hline $60^{0}$ & \multicolumn{1}{c|}{$\frac{15}{8}$ or $\left(2-\frac{1}{8}\right)$} & $19.983^{0}$ approximately \\
\hline 30 & 2 & $10.05^{0}$ approx. \\
\hline 20 & 2 & $6.682^{0}$ approx. \\
\hline 10 & 2 & $3.335^{0}$ approx. \\
\hline 6 & 2 & $2.00043^{0}$ approx. \\
\hline
\end{tabular}

From above if will be seen, when $\theta \rightarrow 0^{0}$, "2"is the limiting value of ' $m$ ' for trisection of any angle.

So the values from $\theta=0^{0}$ to $90^{\circ}, m$ ranges between 2 to $\sqrt{3}$

For ' $m$ ' formula (4) and for $\frac{m}{n}$ formula (5) can be taken to obtain the value of $\tan \psi$ or $\psi$

B. other results-

\begin{tabular}{|c|c|c|c|}
\hline & $\theta$ & $m$ or $\frac{m}{n}$ & $\psi$ \\
\hline \multirow{4}{*}{ Voluntary } & $90^{\circ}$ & $\sqrt{3}$ & $30^{0}$ exactaly \\
\hline & 90 & 1.2 or $\frac{6}{5}$ & $25^{0}$ approximately \\
\hline & 90 & .84 or $\frac{21}{25}$ & $20^{0}$ approx. \\
\hline & 60 & 4.5 or $\frac{9}{2}$ & $25^{0}$ approx. \\
\hline \multirow{3}{*}{ Pentasection } & 90 & $\sqrt{3}-1$ & $18.1^{0}$ approx. \\
\hline & 60 & $\frac{9}{13}$ & 12.003 approx. \\
\hline & 30 & $\frac{2}{3}$ & $5.97^{0}$ \\
\hline
\end{tabular}

3. Special Case: when $\theta=90^{\circ}, \cos \theta=0, \sin \theta=1$ then formula (4)becomes

$\tan \psi=\frac{\sqrt{1+m^{1}}-1}{m}$

put $m=\sqrt{3}$ then

$\tan \psi=\frac{\sqrt{1+3}-1}{\sqrt{3}}$

$=\frac{2-1}{\sqrt{3}}=\frac{1}{\sqrt{3}}=\tan 30^{\circ}$ 
or $\psi=30^{0}=\frac{1}{3}$ of $90^{0}$ which is true as per results of $A \& B$.

Lettan $\psi=\frac{\sqrt{1+m^{2}}-1}{m}=\mathrm{k}$

So $\sqrt{1+m^{2}}-1=m k$

or $\sqrt{1+m^{2}}-1=m k$

or $1+m^{2}=1+m^{2} k^{2}+2 m k$

orm ${ }^{2}-m^{2} k^{2}=2 m k$

$\operatorname{orm}^{2}\left(1-k^{2}\right)=2 m k$

orm $=\frac{2 k}{1-k^{2}}=\tan 2 \psi$

So for given $\mathrm{k}, \mathrm{m}$ can be evaluated exact or approx.for $\theta=90^{\circ}$ and also for any angle $\theta$ applying the results (4) or (5). Nearest fractional values can be obtained by applying continued fractions methods.

For Example if $\theta=90^{\circ}$ then for trisection

$\psi=30^{\circ}$ So by result (9)

$\mathrm{m}=\tan 2 \psi=\tan 2.30^{\circ}$

$=\tan 60^{\circ}=\sqrt{3}$ as in result $\mathrm{A}$

For $\psi=15^{\circ} \mathrm{m}=\tan 2.15^{\circ}$

$=\tan 30=\frac{1}{\sqrt{3}}$

For $\psi=20^{\circ}, \mathrm{m}=\tan 40^{\circ}$

$=.84=\frac{21}{25}$ approximately as in result $\mathrm{B}$

For $\psi=25^{\circ} \mathrm{m}=\tan 50^{\circ}$

$=1.19=\frac{6}{5}$ approximately As in result $\mathrm{B}$

4. How to draw lines equal to

$\mathrm{BD}=\mathrm{mr}$ with the help of unmarked straight edge and compass only.

We know that by simple school level geometry methods any line can be divided into any number of equal parts. This method can be used here.

i) When $\mathrm{m}$ is an integer like $2,3,4,5 \ldots \ldots \ldots$ simply $\mathrm{BD}=\mathrm{m}$ times of radius and can be drawn by compass only.

ii) When $\mathrm{m}=\frac{p}{q}$ a fractional form then $\mathrm{BD}=\frac{p r}{q}=\mathrm{p} \times\left(\frac{r}{q}\right)$ in this cuse $\mathrm{r}$ can be divided into $\mathrm{q}$ equal parts and p part of it will be take to draw BD. All this exercise can be done with the help of straight edge and compass only.

e.g. $\frac{15 r}{8}=\left(1+\frac{7}{8}\right) r=\left(2-\frac{1}{8}\right) r$

$\mathrm{BD}=\left(r+\frac{7}{8} r\right)$ or $\left(2 r-\frac{1}{8} r\right)$ can be draw in parts.

iii) When $\mathrm{m}=\sqrt{p}$ likes $\sqrt{2}, \sqrt{3}, \sqrt{5}$ etc.

Then $\mathrm{BD}=\sqrt{p} \mathrm{r}$ like $\sqrt{2} \mathrm{r}, \sqrt{3} \mathrm{r}$

in right angled triangle Fig. 4

$\mathrm{ABC}$ if $\mathrm{AB}=\mathrm{BC}=\mathrm{r}$

then $A C=\sqrt{2} r$


in fig. $5 \mathrm{rt}$ angled triangle if $\mathrm{AC}=2 \mathrm{r}$

$\mathrm{CB}=\mathrm{r}$ then

$\mathrm{AB}=\sqrt{3} \mathrm{r}$

$$
\sqrt{3} r
$$




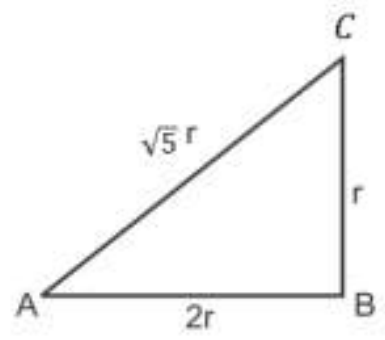

Fig.6

In fig.6

$\mathrm{BC}=\mathrm{r} \quad \mathrm{AB}=2 \mathrm{r}$

Then $\mathrm{AC}=\sqrt{5} \mathrm{r}$

In fig.7

If $\mathrm{AB}=\sqrt{5} \mathrm{r}$

$\mathrm{BC}=\mathrm{r}$

Then $\mathrm{AC}=\sqrt{6} \mathrm{r}$

Similarly any line equal to $\sqrt{p} \mathrm{r}$ can be drawn.

iv) If $\mathrm{m}=\frac{\sqrt{b}}{\sqrt{q}}$

The $\mathrm{BD}=\frac{\sqrt{b}}{\sqrt{q}} \mathrm{r}=\frac{\sqrt{p q}}{q} \mathrm{r}$

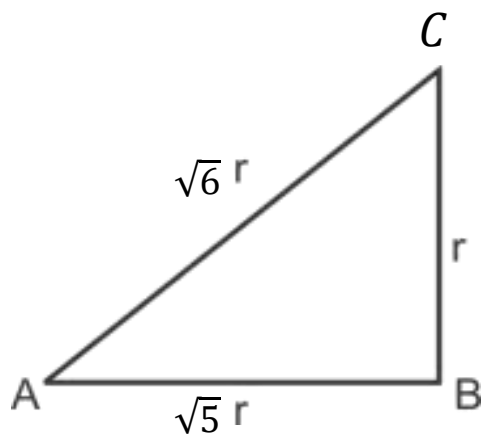

Fig.7

Then $\sqrt{p q} \mathrm{r}$ can be drawn as in (iii) and then divided into q equal parts.

e.q. $\frac{\sqrt{3}}{\sqrt{2}} r=\frac{\sqrt{6}}{2} r$

in fig. (7) $\mathrm{AC}=\sqrt{6} \mathrm{r}$

so $\mathrm{BD}=\frac{\sqrt{6}}{2} \mathrm{r}=\frac{A C}{2}$

v) If $m$ is like $\sqrt{3}-1 \ldots \ldots$.

$\mathrm{BD}=(\sqrt{3}-1) \mathrm{r}$

$=\sqrt{3} \mathrm{r}-\mathrm{r}$ which can be draw n easily so BD can be drawn with the help of straights edge and compass only for all above type of values of $\mathrm{m}$.

5. To find the limiting values of $\mathrm{m}$ when $\boldsymbol{\theta}$ is small and tends to zero

As in result (4) we have

$\tan \psi=\frac{\sqrt{1+m^{2}+2 m \cos \theta}-(1+m \cos \theta)}{m \sin \theta}$

$=\frac{\left\{1+m^{2}+2 m\left(1-\frac{\theta^{2}}{! 2}+\frac{\theta^{4}}{! 4} \ldots \ldots \ldots\right)\right\}^{1 / 2}-\left\{1+m\left(1-\frac{\theta^{2}}{! 2}+\frac{\theta^{4}}{! 4} \ldots \ldots \ldots\right)\right\}}{m\left(\theta-\frac{\theta 3}{! 3}+\frac{\theta 5}{! 5} \ldots \ldots \ldots \ldots .\right)}$

Since $\theta$ is small we can discard $\theta^{3}$ and other higher powers of $\theta$

Then,

$\operatorname{Tan} \psi=\frac{\left\{1+m^{2}+2 m-m \theta^{2}\right\}^{1 / 2}-\left\{1+m-\frac{m \theta^{2}}{2}\right\}}{m \theta}$

$=\frac{\left\{(1+m)^{2}-m \theta^{2}\right\}^{1 / 2}-\left\{1+m-\frac{m \theta^{2}}{2}\right\}}{m \theta}$

$=\frac{\left\{(1+m)^{2}\left(1-\frac{m \theta^{2}}{(1+m)^{2}}\right)\right\}^{1 / 2}-\left\{1+m-\frac{m \theta^{2}}{2}\right\}}{m \theta}$

$=\frac{\left\{(1+m)\left(1-\frac{1 m \theta^{2}}{2(1+m)^{2}}\right)\right\}-\left\{1+m-\frac{m \theta^{2}}{2}\right\}}{m \theta}$ 
$=\frac{\left\{1+m-\frac{m \theta^{2}}{2(1+m)}\right\}-\left\{1+m-\frac{m \theta^{2}}{2}\right\}}{m \theta}$

$=\frac{1+m-1-m+\frac{m \theta^{2}}{2}-\frac{m \theta^{2}}{2(1+m)}}{m \theta^{2} m \theta^{2}}$

$=\frac{\frac{m \theta^{2}}{2}-\frac{m \theta^{2}}{2(1+m)}}{m \theta}$

$=\frac{\frac{m \theta^{2}}{2}\left(1-\frac{1}{1+m}\right)}{m \theta}$

$=\frac{\theta}{2} \times \frac{m}{m+1}$

Now since $\theta$ is small

$\operatorname{Tan} \psi=\psi$

So,

$\psi=\frac{\theta}{2} \times \frac{m}{1+m}$.

This equation gives limiting values of $\mathrm{m}$ for different multisections of $\theta$ when $\theta$ is small

i) For trisection $\psi=\frac{\theta}{3}$

So, $\frac{\theta}{3}=\frac{\theta}{2} \times \frac{m}{1+m}$

or $\frac{1}{3}=\frac{m}{2(\mathrm{Hm})}$

or $3 \mathrm{~m}=2(1+\mathrm{m})$

or $3 \mathrm{~m}-2 \mathrm{~m}=2$

or $\mathrm{m}=2$

So as $\theta$ becomes smaller and smaller 2 is the limiting value of $m$ as shown in result $A$.

ii) For penta section

$\psi=\frac{\theta}{5}$

So $\frac{\theta}{5}=\frac{\theta}{2} \cdot \frac{m}{(1+m)}$

or $5 \mathrm{~m}=2 \mathrm{~m}+2$

or $3 \mathrm{~m}=2$

or $\mathrm{m}=\frac{2}{3}$ is the limiting value of $\mathrm{m}$

Similarity for other multisections limiting values can be derived when $\theta$ goes smaller in general for $\frac{\theta_{\text {th }}}{n}$ section $\mathrm{n} \geq 2$

$\mathrm{m}=\frac{2}{n-2}$ is limiting value

It can be verirfied that these limiting values provide very good approximations for multisections of angles up to $30^{\circ}$.

6. Chart showing limiting values of ' $m$ ' for angle $\theta=90^{\circ}$ and when $\theta$ is small.

\begin{tabular}{|c|c|c|c|}
\hline Multisection & $\boldsymbol{\theta}=\mathbf{9 0}^{\mathbf{0}}$ & $\boldsymbol{\theta}$ is small & Difference/trend \\
\hline$\frac{\theta}{2}$ & $\infty$ & 2 & Nil \\
\hline$\frac{\theta}{3}$ & $\sqrt{3}$ & 1 & 0 - $\sqrt{3} /$ increasing \\
\hline$\frac{\theta}{4}$ & 1 & $\frac{2}{3}$ & $.05984 /$ decreasing \\
\hline$\frac{\theta}{5}$ & $\tan 36^{0}$ & $\frac{1}{2}$ & $.07735 /$ decreasing \\
\hline$\frac{\theta}{6}$ & $\tan 30^{0}$ & $\frac{1}{4}$ & $.07492 /$ do \\
\hline$\frac{\theta}{10}$ & $\tan 18^{0}$ & $\frac{2}{n-2}$ & do \\
\hline$\frac{\theta}{n}$ & $\tan \frac{2 \theta^{0}}{n}$ & $\frac{2}{n}$ & $\frac{\pi-2}{n} / \mathrm{do}$ \\
\hline
\end{tabular}


Ex-1 Given $\angle P A Q=\theta$

\section{SOME EXAMPLES}

Construct an angle equal to $\frac{\theta}{4}$ at point $\mathrm{A}$

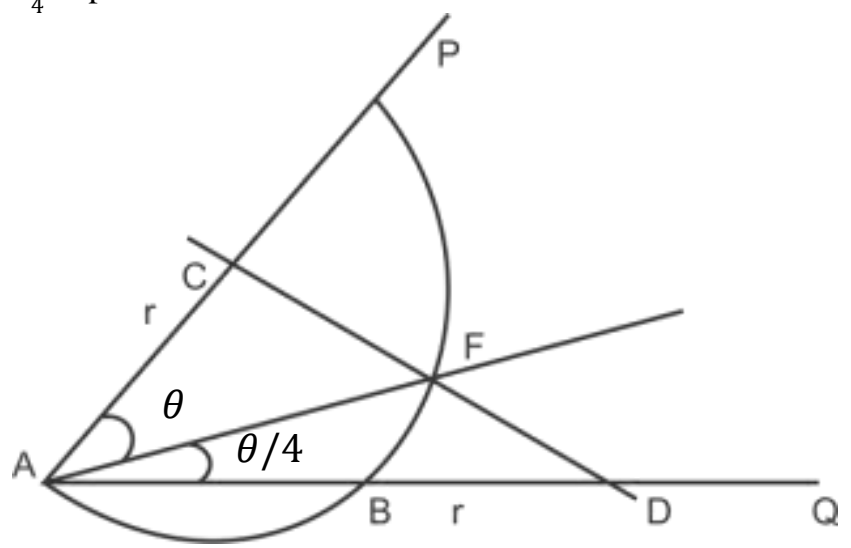

Figure-8

Construction- given $\angle P A Q=\theta$

Take any point $\mathrm{C}$ on $\mathrm{AP}$ and draw a semi circle taking $\mathrm{c}$ as centre and $\mathrm{AC}$ as radius $\mathrm{r}$ which cuts the line $\mathrm{AQ}$ at B.

Cut $\mathrm{BD}=$ radius $\mathrm{r}$ and join ptsC an $\mathrm{D}$ by a line .Let Line $\mathrm{CD}$ cuts the semi circle at $\mathrm{F}$. Then $\angle F A D=\frac{\theta}{4}$

Since here as per corollary-1

$\mathrm{m}=1$ and $\mathrm{BD}=\mathrm{mr}=\mathrm{r}$

nence $\angle F A D=\frac{\theta}{4}$

Ex-2 Given $\angle P A Q=90^{\circ}$, trisect the $\angle P A Q$

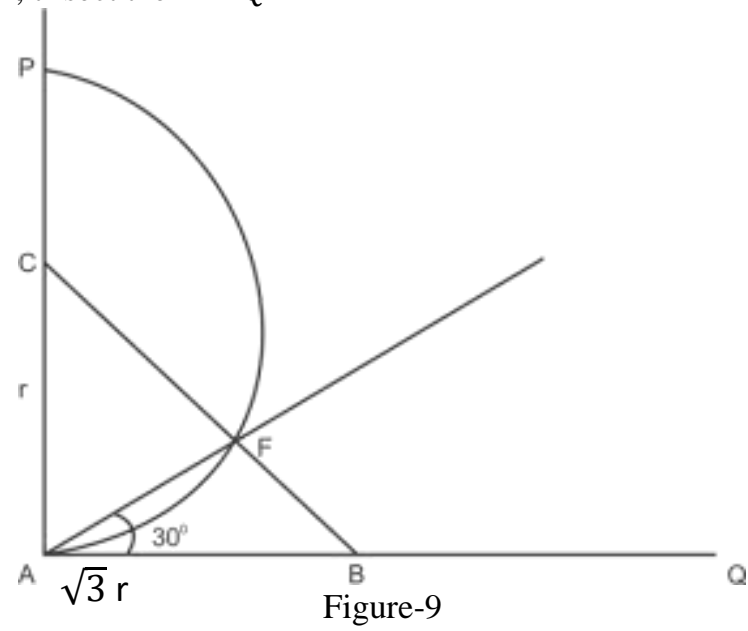

Construction : Given $\angle P A Q=90^{\circ}$

Take $\mathrm{C}$ any point on $\mathrm{AP}$

Draw a semi circle taking $\mathrm{C}$ as centre and $\mathrm{CA}$ as radius (r)

Draw $\mathrm{CB}=2 \mathrm{r}$ by compass $(2 \mathrm{r}=$ diameter of the circle $)$

Point $\mathrm{B}$ being on the line $\mathrm{AQ}$

Let the line $\mathrm{CB}$ cuts the semi circle on $\mathrm{F}$ join $\mathrm{AF}$. Then $\angle F A B=90^{\circ} / 2=30^{\circ}$

By Pythagoras theonem if $\mathrm{AC}=\mathrm{r} ; \mathrm{CB}=2 \mathrm{r}$

$\mathrm{AB}=\sqrt{3} \mathrm{r}$ so here $\mathrm{m}=\sqrt{3}$

By corollary 3 and formula (9) we have

if $\theta=90^{\circ}$ then for $\frac{\theta}{3} \psi=30^{\circ}$

$\mathrm{m}=\tan 2 \psi$

$=\tan 2.30$

$=\tan 60=\sqrt{3}$

Hence $\angle F A B=30^{\circ}$ 
Ex-3 Given $\angle \mathrm{PAQ}=20^{0}$

taking $\mathrm{m}=2$ trisect it and find the value of $\tan \psi \& \psi$ approximately

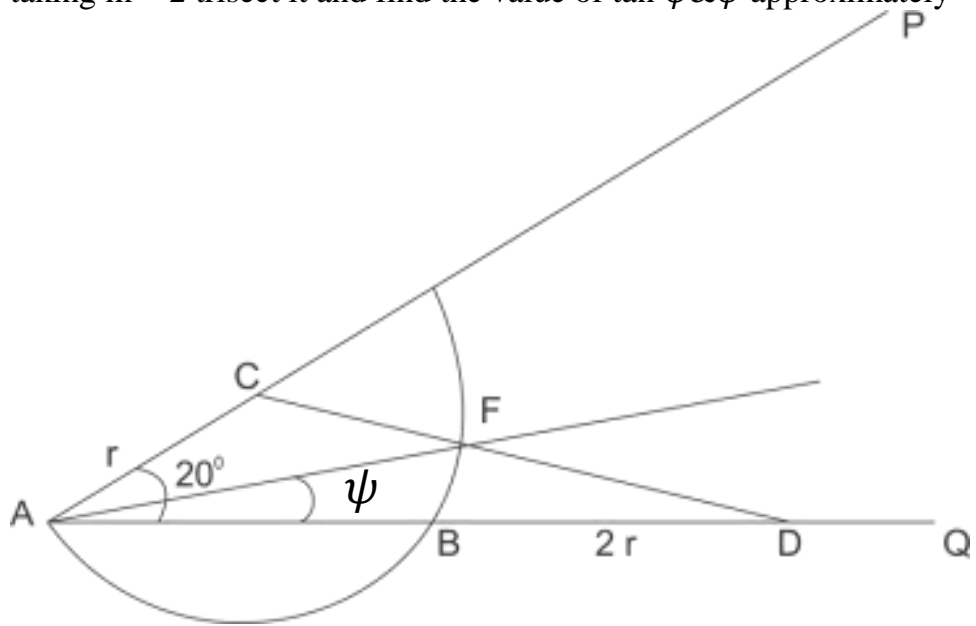

Figure-10

Given $\angle \mathrm{PAQ}=20^{\circ}, \mathrm{m}=2$ so $\mathrm{BD}=2 \mathrm{r}$

$$
\angle \mathrm{FAB}=\psi
$$

Now from formula (4) we have

$\operatorname{Tan} \psi$

$$
\begin{aligned}
& =\quad \frac{\sqrt{1+m^{2}+2 m \cos \theta}-(1+m \cos \theta)}{m \sin \theta} \\
& =\frac{\sqrt{1+2^{2}+2.2 \cos 20^{0}}-\left(1+2 \cos 20^{0}\right)}{2 \sin 20^{0}} \\
& =\frac{\sqrt{5+4 \times .93969262}-(1+2 \times .93969262)}{2 \times .34202014} \\
& =\quad \frac{2.95952200-2.87938524}{.68404028} \\
& =\quad .117152107=\tan 6.68186^{0}
\end{aligned}
$$

So. $\psi=6.68186^{\circ}=\frac{20^{0}}{3}$ approximately

With a difference of $\left(\frac{20}{3}-6.68186\right)$

So $\mathrm{m}=2$ approximately trisects $20^{\circ}$ angle

$$
=\quad-.015193^{\circ} ; \text { slightly higher than } \frac{20^{0}}{3}
$$

Also for $\mathrm{m}=2 ; \theta$ is $30^{\circ}, \psi=10.05^{\circ}$ with a slight difference than $10^{\circ}$ i.e. approximately trisects $30^{\circ}$.

Note: when angle $\theta$ is $>30^{\circ}$ and $<90^{\circ}$

Than for trisection of angle $\theta$ following steps can be adopted.

i) $\quad$ Draw $\frac{1}{4}$ of $\theta$ using corollary 1

ii) Trisect $\frac{\theta}{4}$ to find $\frac{\theta}{12}$ taking $\mathrm{m}=2$

iii) Make an angle $4 \times \frac{\theta}{12}$ i,e 4 limes of the angle obtained in step (ii) this will give very near approximation of $\frac{\theta}{3}$.

Ex-4 Trisect $80^{\circ}$ angle

Step:

i) $\quad \operatorname{Draw} \frac{80}{4}=20^{\circ}$

ii) Trisect $20^{\circ}$ as in Ex-3

iii) Make angle 4 times of (ii)

$$
\begin{array}{lll}
\text { Thus } \psi= & \left\{\left(\frac{1}{4} \times 80\right) \times \frac{1}{3}\right\} \times 4 \\
& = & 6.68186 \times 4 \\
& = & 26.727^{0}=\frac{80}{3} \text { approximately } \\
\text { Defference } & = & 26.727-26.667 \\
& = & .06^{0}
\end{array}
$$

Ex-5 Given angle $\theta=10^{\circ}$

draw $\frac{\theta}{5}$

solution: since $\theta=10^{\circ}$ is a small angle using the formula (11) 
so by formula (5)

$$
\mathrm{m} \quad=\frac{2}{5-2}=\frac{2}{3}
$$

we have

$\tan \psi=\frac{\sqrt{m^{2}+n^{2}+2 m n \cos \theta}-(n+m \cos \theta)}{m \sin \theta}$

putting $\mathrm{m}=2, \mathrm{n}=3$

$\begin{array}{rll}\tan \psi & =\frac{\sqrt{2^{2}+3^{2}+2.23 \cos 10}-(3+2 \cos 10)}{2 \sin 10} \\ & =\quad \frac{\sqrt{13+12 \times \cos 10}-(3+2 \cos 10)}{2 \sin 10} \\ & = & \frac{.01212044}{.34729636} \\ & = & .034899416 \\ & = & \tan 1.998778^{0} \\ & = & \end{array}$

So $\mathrm{m}=\frac{2}{3}$ gives very near approximation of pentasection of $10^{\circ}$ angle $\left(\frac{10^{0}}{5}\right)$ and can be drawn as in prev. examples.

Note: all the construction of angle multisection given in the previous examples can be done with the help of straight edge and compass only.

Reference:

(i) Ordinary geometry, aljebra, coordinate geometry and continued fractions.

(ii) Internet searches. 